\title{
Post-outburst polarimetry of the binary HD 5980 (O4-6/LBV +WN3): rapid density enhancements along the polar axis and equatorial plane
}

\author{
Alfredo Villar-Sbaffi, Anthony F.J. Moffat, and Nicole St-Louis \\ Département de physique, Université de Montréal, \\ C.P. 6128, Succ. Centre-Ville, Montréal, QC H3C 3J7, Canada
}

\begin{abstract}
In 2001, we carried out a series of polarimetric observations of the SMC Wolf-Rayet binary HD 5980 near secondary eclipse, in order to study the mass-outflow of the system. Our observations revealed the presence of intense density enhancements along the poles and equatorial plane, that fluctuate on a very short timescale.
\end{abstract}

\section{Introduction}

When Moffat et al. (1998, hereafter M98) presented the first broadband polarimetric study of the eclipsing Wolf-Rayet binary HD 5980 (O4-6/LBV+WN3) in its pre-outburst phase, they noticed a strange behaviour in the standard equatorial Stokes $Q$ and $U$ parameters at phase 0.36 (when the WR component eclipses the O/LBV component). In order to provide new insight on the post-outburst system and to better understand the source of the unexpected polarimetric variations, we present here the first results from our five nights spent at the Complejo Astronomico El Leoncito (CASLEO), using the CasProf photopolarimeter.

\section{Results}

From our observations we draw the following four conclusions:

(i): a high degree of stochastic polarimetric variability occurs around secondary eclipse. This variability was largest on our last night, reaching an amplitude of more than $1 \%$;

(ii): there is a general trend for a relatively high level of variability in $Q$ and $U$, centered around $0 \%$ the first night to slightly negative values in $Q$ by the end of the night and remaining that way, with a lesser degree of activity, during our second night. The system then entered a period of very high variability on our third and fifth nights, with $Q$ starting to increase towards positive values while $U$ remained centered around $0 \%$;

(iii): on our last night, the de-rotated results show that the electron density had an axial symmetry, since $U$ remained (almost) constant at $\sim 0 \%$. The polarimetric variability in that last night was dominated by matter enhancements along the orbit plane (i.e., $Q>0 \%$ ), although a few points showed polar en- 

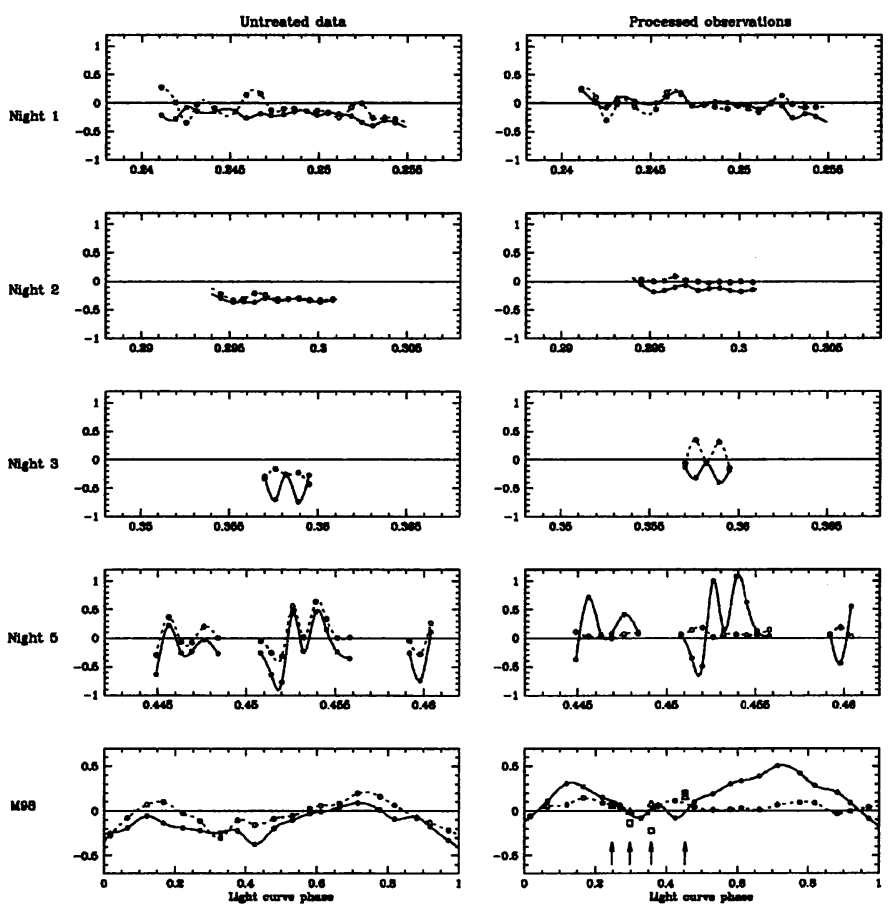

Figure 1. Normalized $Q$ (solid) and $U$ (dashed) parameters connected using a cubic spline function. The processed results have been de-rotated to the orbital plane of symmetry and have their interstellar polarization and intrinsic BME variability removed (Brown et al. 1982; Simons \& Boyle 1984; Robert et al. 1992). On the right hand bottom corner, the arrows indicate the times of our observations, the empty squares are the average $Q$ values and the empty triangles are the average $U$ values for each of our nights.

hancements $(Q<0 \%)$ at a lower amplitude $(\sim 0.4 \%)$. This rapid variability occurred on a very short time scale of a few minutes; and

(iv): by superimposing our averaged results for each night together with the binned data of M98, we can see that the $U$ parameter has remained constant since 1990, while the $Q$ parameter seems to deviate from the pre-outburst value. This strongly suggests that the density along the equatorial plane and polar axis has increased since 1987-90.

\section{References}

Brown, J.C., Aspin, C., Simmons, J.F.L., McLean, I.S. 1982, MNRAS 198, 787

Moffat, A.F.J., Marchenko, S.V., Bartzakos, P., et al. 1998, ApJ 497, 896

Robert, C., Moffat, A.F.J., Drissen, L., et al. 1992, ApJ 397, 277

Simmons, J.F.L., Boyle, C.B. 1984, A\&A 134, 368 